quatrième série-tome 43 fascicule 6 novembre-décembre 2010

$$
\begin{aligned}
& \text { ANNALES } \\
& \text { SCIENTIFIQUES } \\
& \text { de } \\
& \text { L'ECOLE } \\
& \text { NORMALE } \\
& \text { SUPÉRIEURE }
\end{aligned}
$$

\author{
Qëndrim R. GASHI \\ On a conjecture of Kottwitz. and Rapoport
}


Ann. Scient. Éc. Norm. Sup.

$4^{\mathrm{e}}$ série, t. 43,2010 , p. 1017 à 1038

\title{
ON A CONJECTURE OF KOTTWITZ AND RAPOPORT
}

\author{
BY QËNDRIM R. GASHI
}

To Bob Kottwitz, my dedicated teacher and mentor, with profound gratitude and admiration.

Abstract. - We prove a conjecture of Kottwitz and Rapoport which implies a converse to Mazur's Inequality for all (connected) split and quasi-split unramified reductive groups. Our results are related to the non-emptiness of certain affine Deligne-Lusztig varieties.

RÉSUmÉ. - On démontre une conjecture de Kottwitz et Rapoport sur une réciproque à l'inégalité de Mazur pour tout groupe (connexe) réductif, déployé ou quasi-déployé non-ramifié. Nos résultats sont liés à la non-vacuité de certaines variétés de Deligne-Lusztig affines.

\section{Introduction}

Mazur's Inequality ([14], [15]) is related to the study of $p$-adic estimates of the number of points of certain algebraic varieties over a finite field of characteristic $p$. It is most easily stated using isocrystals. Before stating the precise inequality, we recall the definition of an isocrystal: it is a pair $(V, \Phi)$, where $V$ is a finite-dimensional vector space over the fraction field $K$ of the ring of Witt vectors $W\left(\overline{\mathbb{F}}_{p}\right)$, and $\Phi$ is a $\sigma$-linear bijective endomorphism of $V$, where $\sigma$ is the automorphism of $K$ induced by the Frobenius automorphism of $\overline{\mathbb{F}}_{p}$. Next, we recall Mazur's inequality.

Suppose that $(V, \Phi)$ is an isocrystal of dimension $n$. By Dieudonné-Manin theory, we can associate to $V$ its Newton vector

$$
\nu(V, \Phi) \in\left(\mathbb{Q}^{n}\right)_{+}:=\left\{\left(\nu_{1}, \ldots, \nu_{n}\right) \in \mathbb{Q}^{n}: \nu_{1} \geq \nu_{2} \geq \cdots \geq \nu_{n}\right\}
$$

which classifies isocrystals of dimension $n$ up to isomorphism. If $\Lambda$ is a $W\left(\overline{\mathbb{F}}_{p}\right)$-lattice in $V$, then we can associate to $\Lambda$ the Hodge vector $\mu(\Lambda) \in\left(\mathbb{Z}^{n}\right)_{+}:=\left(\mathbb{Q}^{n}\right)_{+} \cap \mathbb{Z}^{n}$, which measures the relative position of the lattices $\Lambda$ and $\Phi(\Lambda)$. Let $\nu(V, \Phi):=\left(\nu_{1}, \ldots, \nu_{n}\right)$ and $\mu(\Lambda):=\left(\mu_{1}, \ldots, \mu_{n}\right)$. Mazur's Inequality asserts that $\mu(\Lambda) \geq \nu(V, \Phi)$, where $\geq$ is the 
dominance order, i.e., $\mu_{1} \geq \nu_{1}, \mu_{1}+\mu_{2} \geq \nu_{1}+\nu_{2}, \cdots, \mu_{1}+\ldots \mu_{n-1} \geq \nu_{1}+\ldots \nu_{n-1}$, and $\mu_{1}+\ldots \mu_{n}=\nu_{1}+\ldots \nu_{n}$.

A converse to this inequality is proved by Kottwitz and Rapoport in [11], where they show that if $(V, \Phi)$ is an isocrystal of dimension $n$ and $\mu \in\left(\mathbb{Z}^{n}\right)_{+}$is such that $\mu \geq \nu(V, \Phi)$, then there exists a $W\left(\overline{\mathbb{F}}_{p}\right)$-lattice $\Lambda$ in $V$ satisfying $\mu=\mu(\Lambda)$.

Both Mazur's Inequality and its converse can be regarded as statements for the group $G L_{n}$, since the dominance order arises naturally in the context of the root system for $G L_{n}$. In fact, there is a bijection (see [9]) between isomorphism classes of isocrystals of dimension $n$ and the set of $\sigma$-conjugacy classes in $G L_{n}(K)$. Kottwitz studies in ibid. the set $B(G)$ of the $\sigma$-conjugacy classes in $G(K)$, for a connected reductive group $G$ over $\mathbb{Q}_{p}$, and, as he notes, there is a bijection between $B(G)$ and the isomorphism classes of isocrystals with " $G$-structure" of a certain dimension (for $G=G L_{n}$ these are simply the above isocrystals). Thus, results on isocrystals, and more generally isocrystals with additional structure, are related to those on the $\sigma$-conjugacy classes of certain reductive groups.

With this viewpoint in mind, we are interested in the group-theoretic generalizations of Mazur's Inequality and its converse, especially since they appear naturally in the study of the non-emptiness of certain affine Deligne-Lusztig varieties. To make these statements more precise, we introduce some notation.

Let $F$ be a finite extension of $\mathbb{Q}_{p}$, with uniformizing element $\pi$, and let $\mathfrak{o}_{F}$ be the ring of integers of $F$. Suppose that $G$ is a split connected reductive group over $F$ (unramified quasi-split groups are treated in the last section of the paper). Let $B$ be a Borel subgroup in $G$ and $T$ a maximal torus in $B$, both defined over $\mathfrak{o}_{F}$. Let $L$ be the completion of the maximal unramified extension of $F$ in some algebraic closure of $F$, and $\sigma$ the Frobenius automorphism of $L$ over $F$. The valuation ring of $L$ is denoted by $\mathfrak{o}_{L}$.

We write $X$ for the group of co-characters $X_{*}(T)$. Let $\mu \in X$ be a dominant element and $b \in G(L)$. The affine Deligne-Lusztig variety $X_{\mu}^{G}(b)$ is defined by

$$
X_{\mu}^{G}(b):=\left\{x \in G(L) / G\left(\mathfrak{o}_{L}\right): x^{-1} b \sigma(x) \in G\left(\mathfrak{o}_{L}\right) \mu(\pi) G\left(\mathfrak{o}_{L}\right)\right\} .
$$

These $p$-adic "counterparts" of the classical Deligne-Lusztig varieties get their name by virtue of being defined in a similar way as the latter, and have been studied by a number of authors (see, for example, [8], [7], [23], and references therein). For the relevance of affine Deligne-Lusztig varieties to Shimura varieties, the reader may wish to consult [18].

We need some more notation to be able to formulate the group-theoretic generalizations of Mazur's Inequality and its converse. Let $P=M N$ be a parabolic subgroup of $G$ that contains $B$, where $M$ is the unique Levi subgroup of $P$ containing $T$. The Weyl group of $T$ in $G$ is denoted by $W$. We let $X_{G}$ and $X_{M}$ be the quotient of $X$ by the coroot lattice for $G$ and $M$, respectively. Also, we let $\varphi_{G}: X \rightarrow X_{G}$ and $\varphi_{M}: X \rightarrow X_{M}$ denote the respective natural projection maps.

Let $B=T U$, with $U$ the unipotent radical. If $g \in G(L)$, then there is a unique element of $X$, denoted by $r_{B}(g)$, so that $g \in G\left(\mathfrak{o}_{L}\right) r_{B}(g)(\pi) U(L)$. We have a well-defined map $w_{G}: G(L) \rightarrow X_{G}$, the Kottwitz map [9], where for $g \in G(L)$, we write $w_{G}(g)$ for the image of $r_{B}(g)$ under the canonical surjection $X \rightarrow X_{G}$. In a completely analogous way, considering $M$ instead of $G$, one defines the map $w_{M}: M(L) \rightarrow X_{M}$. 
We use the partial ordering $\stackrel{P}{\leq}$ in $X_{M}$, where for $\mu, \nu \in X_{M}$, we write $\nu \stackrel{P}{\leq} \mu$ if and only if $\mu-\nu$ is a nonnegative integral linear combination of the images in $X_{M}$ of the coroots corresponding to the simple roots of $T$ in $N$.

We will make use of a subset $X_{M}^{+}$of $X_{M}$, which we now define. Let $\mathfrak{a}_{P}:=X_{*}\left(T_{P}\right) \otimes_{\mathbb{Z}} \mathbb{R}$, where $T_{P}$ is the identity component of the center of $M$ (and thus $T_{P}$ is a split torus over $F$ ). Note that there is a canonical isomorphism $\varrho: \mathfrak{a}_{P} \simeq X_{M} \otimes_{\mathbb{Z}} \mathbb{R}$ obtained by tensoring with $\mathbb{R}$ the composition $X_{*}\left(T_{P}\right) \hookrightarrow X_{*}(T) \rightarrow X_{M}$. Let $\xi: X_{M} \rightarrow X_{M} \otimes_{\mathbb{Z}} \mathbb{R}$ be the natural map. The subset $X_{M}^{+} \subset X_{M}$ is defined as the set of all elements $\nu \in X_{M}$ such that $\left(\varrho^{-1} \circ \xi\right)(\nu)$ lies in the subset

$$
\left\{x \in \mathfrak{a}_{P}:\langle\alpha, x\rangle>0, \text { for every root } \alpha \text { of } T_{P} \text { in } N\right\} \subset \mathfrak{a}_{P} .
$$

The pairing $\langle$,$\rangle appearing in the last line is induced by the usual one between weights and$ coweights of $T_{P}$.

Next, let $b \in M(L)$. We recall briefly the notion of $b$ being basic (for further details see [9]): In loc. cit., $\S 4$, Kottwitz defines a map $\epsilon: M(L) \rightarrow \operatorname{Hom}_{L}(\mathbb{D}, M)$, which he denotes by $\nu$, and where $\mathbb{D}$ is the diagonalizable pro-algebraic group over $\mathbb{Q}_{p}$ whose character group is $\mathbb{Q}$. An element $b \in M(L)$ is called basic if $\epsilon(b) \in \operatorname{Hom}_{L}(\mathbb{D}, M)$ factors through the center of $M$. The element $\epsilon(b)$ is linked with the slopes of the isocrystal corresponding to $b$. Let us mention that $\epsilon(b)$ is characterized by the existence of an integer $n>0$, an element $c \in M(L)$ and a uniformizing element $\pi$ of $F$ such that the following three conditions hold:

$$
\begin{gathered}
n \epsilon(b) \in \operatorname{Hom}_{L}\left(\mathbb{G}_{m}, M\right), \\
\operatorname{Int}(c) \circ(n \epsilon(b)) \text { is defined over the fixed field of } \sigma^{n} \text { on } L, \text { and } \\
c(b \sigma)^{n} c^{-1}=c \cdot(n \epsilon(b))(\pi) \cdot c^{-1} \cdot \sigma^{n},
\end{gathered}
$$

where $\operatorname{Int}(c)$ denotes the inner automorphism $x \mapsto c x c^{-1}$ of $M(L)$, and where we recall that $\sigma$ is the Frobenius of $L$ over $F$.

We now state the first main result of this paper.

Theorem 1.1. - Let $\mu \in X$ be dominant and let $b \in M(L)$ be a basic element such that $w_{M}(b)$ lies in $X_{M}^{+}$. Then

$$
X_{\mu}^{G}(b) \neq \varnothing \Longleftrightarrow w_{M}(b) \stackrel{P}{\leq} \varphi_{M}(\mu)
$$

We prove a similar theorem for quasi-split unramified groups. The precise formulation (Theorem 5.1) and the proof of that result is postponed until the last section of the paper.

We remark that since every $\sigma$-conjugacy class in $G(L)$ contains an element that is basic in some standard Levi subgroup $M$ (see [9]), Theorem 1.1 proves the non-emptiness of the affine Deligne-Lusztig varieties $X_{\mu}^{G}(b)$, where $b \in G(L)$.

One direction in the theorem, namely

$$
X_{\mu}^{G}(b) \neq \varnothing \Longrightarrow w_{M}(b) \stackrel{P}{\leq} \varphi_{M}(\mu)
$$

is the group-theoretic generalization of Mazur's Inequality, and it is proved by Rapoport and Richartz in [19] (see also [10, Theorem 1.1, part (1)]). 
The other direction, i.e., the group-theoretic generalization of the converse to Mazur's Inequality, is a conjecture of Kottwitz and Rapoport [11]. Next, we discuss how their conjecture is reduced to one formulated only in terms of root systems. Let

$$
\mathscr{P}_{\mu}:=\left\{\nu \in X:(\mathrm{i}) \varphi_{G}(\nu)=\varphi_{G}(\mu) \text {; and (ii) } \nu \in \operatorname{Conv}(W \mu)\right\},
$$

where $\operatorname{Conv}(W \mu)$ is the convex hull of the Weyl orbit $W \mu:=\{w(\mu): w \in W\}$ of $\mu$ in $\mathfrak{a}:=X \otimes_{\mathbb{Z}} \mathbb{R}$. Then we have (cf. [10, Theorem 4.3])

$$
X_{\mu}^{G}(b) \neq \varnothing \Longleftrightarrow w_{M}(b) \in \varphi_{M}\left(\mathscr{P}_{\mu}\right) .
$$

Thus the other implication in Theorem 1.1 follows if we show that

$$
w_{M}(b) \stackrel{P}{\leq} \varphi_{M}(\mu) \Longrightarrow w_{M}(b) \in \varphi_{M}\left(\mathscr{D}_{\mu}\right) .
$$

For this, it suffices to show that for $\nu \in X_{M}$ we have

$$
\nu \stackrel{P}{\leq} \varphi_{M}(\mu) \Longrightarrow \nu \in \varphi_{M}\left(\mathscr{D}_{\mu}\right) \text {. }
$$

(Note that the condition from Theorem 1.1 that $b \in M(L)$ be basic does not appear in the last implication. Also, we do not require that $\nu \in X_{M}^{+}$, but only that $\nu \in X_{M}$.) As can be seen from [10, Section 4.4], we have

$$
\nu \stackrel{P}{\leq} \varphi_{M}(\mu) \Longleftrightarrow\left\{\begin{array}{l}
\text { (i) } \nu \text { and } \mu \text { have the same image in } X_{G}, \text { and } \\
\text { (ii) the image of } \nu \text { in } \mathfrak{a}_{M} \text { lies in } \operatorname{pr}_{M}(\operatorname{Conv}(W \mu)) .
\end{array}\right.
$$

Taking into account (2), the implication (1) can be reformulated:

$$
\left.\begin{array}{l}
\text { (i) } \nu \text { and } \mu \text { have the same image in } X_{G} \text {, and } \\
\text { (ii) the image of } \nu \text { in } \mathfrak{a}_{M} \text { lies in } \operatorname{pr}_{M}(\operatorname{Conv}(W \mu))
\end{array}\right\} \Longrightarrow \nu \in \varphi_{M}\left(\mathscr{P}_{\mu}\right) \text {. }
$$

The implication (3) follows from

TheOREM 1.2 (Kottwitz-Rapoport Conjecture; split case). - We have that

$$
\begin{aligned}
\varphi_{M}\left(\mathscr{P}_{\mu}\right)=\left\{\nu \in X_{M}\right. & : \text { (i) } \nu, \mu \text { have the same image in } X_{G} ; \\
& \text { (ii) the image of } \left.\nu \text { in } \mathfrak{a}_{M} \text { lies in } \operatorname{pr}_{M}(\operatorname{Conv}(W \mu))\right\},
\end{aligned}
$$

where $\mathfrak{a}_{M}:=X_{M} \otimes_{\mathbb{Z}} \mathbb{R}$ and $\operatorname{pr}_{M}: \mathfrak{a} \rightarrow \mathfrak{a}_{M}$ denotes the natural projection induced by $\varphi_{M}$.

For the above theorem, it is easily seen that the set on the left-hand side is contained in the set on the right-hand side. The point is to prove the converse, which is equivalent to the implication (3).

A variant of Theorem 1.2, in the case of quasi-split unramified groups, is proved in the last section (see Theorem 5.2). We remark that Theorem 1.2 is a statement that is purely a root-theoretic one, so it remains true when we work over other fields of characteristic zero, not just $\mathbb{Q}_{p}$.

Theorem 1.2 had been previously proved for $G L_{n}$ and $G S p_{2 n}$ by Kottwitz and Rapoport [11] and then for all classical groups by Lucarelli [12]. In addition, Wintenberger, using different methods, proved this result for $\mu$ minuscule (see [24]). A more general version of this theorem for $G L_{n}$ was proved in [5, Theorem A] using the theory of toric varieties. (For more details about the precise relation between Theorem 1.2 and cohomology-vanishing on toric varieties associated with root systems see [5], [4].) 
At the end of this introduction, let us describe how our paper is organized. In Section 2 we prove Theorem 1.2 in the case of simply-laced root systems (i.e., root systems of type $A, D$, or $E$ ). Some auxiliary results used in this proof are treated in the next section. An interesting feature of the proof of Theorem 1.2 is that its last part involves Peterson's notion of minuscule Weyl group elements (cf. [22]) or, equivalently, the numbers game with a cutoff [6] — this is a modified version of the so-called Mozes' game of numbers (cf. [16]). Section 4 is devoted to the proof of Theorem 1.2 in the case of non-simply laced root systems, where we use a folding argument to deduce the result from the analogous statement for the simply-laced one. The last section contains the proof of a converse to Mazur's Inequality for unramified quasi-split groups.

Acknowledgments. - It is with special pleasure and great gratitude that we thank Robert Kottwitz, to whom this paper is dedicated, for his time, invaluable advice and comments, and for carefully reading earlier versions of this paper. We heartily thank Travis Schedler for allowing the inclusion in this paper of joint results appearing on Section 3 and for very fruitful discussions on the numbers game. We also thank Michael Rapoport and Ulrich Görtz for comments on an earlier version of this paper, and Eva Viehmann for helpful conversations. We thank Artan Berisha for help with a computer program. Part of this work was supported by an EPDI Fellowship and a Clay Liftoff Fellowship. We thank the University of Chicago and the Max Planck Institute of Mathematics in Bonn for their hospitality.

\section{The case of simply-laced root systems}

Since the statement of Theorem 1.2 only involves root systems and since we will be using facts from [2], we shall rewrite the statement of our main result so that it conforms to the notation from [2]. Moreover, we will be working with roots, instead of coroots (which can also be interpreted to mean that we will be working with the Langlands' complex dual group of $G$, instead of with the group $G$ from the introduction).

Suppose that $R$ is a reduced, irreducible root system and $W$ is its Weyl group. Denote by $P(R)$ and $Q(R)$ the weight and radical-weight lattices for $R$, respectively. Let $\Delta:=\left\{\alpha_{i}: i \in I\right\}$, where $I:=\{1, \ldots, n\}$, be the simple roots (for some choice) in $R$. Let $\varnothing \neq J \varsubsetneqq I$ and consider the sub-root system, denoted by $R_{J}$, corresponding to the set of simple roots $\left\{\alpha_{j}: j \in J\right\}$ (this corresponds to the Levi group $M$ from the introduction). Let $Q\left(R_{J}\right)$ be defined similarly to $Q(R)$.

Let $\mu \in P(R)$ be a dominant weight, i.e., $\left\langle\mu, \alpha_{i}^{\vee}\right\rangle \geq 0, \forall i \in I$, where $\alpha_{i}^{\vee}$ is the coroot corresponding to $\alpha_{i}$, and $\langle$,$\rangle stands for the canonical pairing between weights and$ coweights of $R$. Let $\varphi$ and $\varphi_{J}$ be the natural projections of $P(R)$ onto $P(R) / Q(R)$ and onto $P(R) / Q\left(R_{J}\right)$, respectively. Consider the convex hull $\operatorname{Conv}(W \mu) \subset P(R) \otimes_{\mathbb{Z}} \mathbb{R}$, of the Weyl orbit of $\mu$. Recall that we defined

$$
\mathscr{P}_{\mu}:=\{\nu \in P(R):(\mathrm{i}) \varphi(\nu)=\varphi(\mu) \text {; and (ii) } \nu \in \operatorname{Conv}(W \mu)\} .
$$

If we write $\operatorname{pr}_{J}$ for the natural projection

$$
P(R) \otimes_{\mathbb{Z}} \mathbb{R} \rightarrow\left(P(R) / Q\left(R_{J}\right)\right) \otimes_{\mathbb{Z}} \mathbb{R},
$$


induced by $\varphi_{J}$, then Theorem 1.2 can be reformulated as follows.

THeOREM 2.1. - We have that $\varphi_{J}\left(\mathscr{P}_{\mu}\right)=\left\{y \in P(R) / Q\left(R_{J}\right):(\mathrm{i}) y, \mu\right.$ have the same image in $P(R) / Q(R)$;

(ii) the image of $y$ in $\left(P(R) / Q\left(R_{J}\right)\right) \otimes_{\mathbb{Z}} \mathbb{R}$ lies in $\left.\operatorname{pr}_{J}(\operatorname{Conv}(W \mu))\right\}$.

Note that to prove Theorem 2.1 it is sufficient to prove that the right-hand side is contained in the left-hand side, since the converse is clear.

Suppose that $y$ is an element of the set appearing on the right-hand side in Theorem 2.1. We may consider $y$ as an element of $P(R) \otimes_{\mathbb{Z}} \mathbb{R}$. Indeed, consider the short exact sequence

$$
\operatorname{ker}\left(\varphi_{J}\right) \hookrightarrow P(R) \stackrel{\varphi_{J}}{\rightarrow} P(R) / Q\left(R_{J}\right)
$$

Tensoring by $\mathbb{R}$ any torsion is lost, thus we may consider $\left(P(R) / Q\left(R_{J}\right)\right) \otimes_{\mathbb{Z}} \mathbb{R}$ as a subspace of $P(R) \otimes_{\mathbb{Z}} \mathbb{R}$, where the elements of the former are orthogonal to the coroots of $R_{J}$, with respect to the canonical pairing $\langle$,$\rangle . So, we may identify y$ with the element $y \otimes 1 \in\left(P(R) / Q\left(R_{J}\right)\right) \otimes_{\mathbb{Z}} \mathbb{R} \subset P(R) \otimes_{\mathbb{Z}} \mathbb{R}$. Moreover, without loss of generality, we assume that $y$ is dominant.

There exists a unique element $z \in P(R)$ which is $J$-minuscule, $J$-dominant and such that $\operatorname{pr}_{J}(z)=y$ (cf. [3, Ch. VIII, §7, Proposition 8]). We recall that $z$ being $J$-minuscule means that $\left\langle z, \alpha^{\vee}\right\rangle \in\{-1,0,1\}$, for all roots $\alpha$ in $R_{J}$, and $z$ being $J$-dominant means that $\left\langle z, \alpha_{j}^{\vee}\right\rangle \geq 0, \forall j \in J$. If we do not modify the adjectives dominant and minuscule, then they will always mean $I$-dominant and $I$-minuscule. Also, note that the notions of $J$ or $I$-minusculity are similarly defined for elements of $P(R) \otimes_{\mathbb{Z}} \mathbb{R}$ other than $P(R)$.

Now identifying $z$ with $z \otimes 1 \in P(R) \otimes_{\mathbb{Z}} \mathbb{R}$, we can write

$$
z=y+\sum_{j \in J} k_{j} \alpha_{j}
$$

for some non-negative reals $k_{j}$. Instead of $z$, consider its " $J$-fractional part"

$$
z^{\prime}:=y+\sum_{j \in J} k_{j}^{\prime} \alpha_{j}
$$

where, for each $j, k_{j}^{\prime}$ stands for the fractional part of $k_{j}$. Clearly, $\operatorname{pr}_{J}\left(z^{\prime}\right)=y$. Then Theorem 2.1 follows from the following result.

Proposition 2.2. - The element $z^{\prime}$ lies in $\mathscr{P}_{\mu}$.

We should mention here that a similar proposition, in the case of classical groups, was proved in [12], but there $z$ was shown to lie in $\mathscr{P}_{\mu}$ and $z^{\prime}$ was not considered at all. For our proof, as will become apparent shortly, it is essential that we consider $z^{\prime}$ instead of $z$. A priori, we do not know that $z$ lies in $\mathscr{P}_{\mu}$. However, it turns out that (at least for root systems of type $A, D$, and $E$ ) the elements $z$ and $z^{\prime}$ are in the same Weyl orbit (Proposition 3.1), and therefore $z$ also lies in $\mathscr{P}_{\mu}$.

Since we have assumed that $\mu$ and $y$ have the same image in $P(R) / Q(R)$, we immediately get that $\mu$ and $z^{\prime}$ also have the same image in $P(R) / Q(R)$. Thus, to prove Proposition 2.2, we only need to show that $z^{\prime} \in \operatorname{Conv}(W \mu)$, which will indeed occupy the rest of this section.

$4^{\mathrm{e}}$ SÉRIE - TOME $43-2010-\mathrm{N}^{\mathrm{o}} 6$ 
Before we start with some auxiliary results, let us make an important assumption. We will assume that $R$ is a simply-laced root system, i.e., the roots of $R$ have equal length, or equivalently, the root system $R$ is of one of the types: A, D or E. The result of Theorem 2.1 for the non-simply laced root systems will follow from the analogous result for the simplylaced root systems by the well-known argument of folding. This is carried out in Section 4. The reason why we first consider only root systems that are simply-laced is given in Remark 2.4 below.

Recall (see [1, Lemma 3.1]) that a dominant element $x \in P(R) \otimes_{\mathbb{Z}} \mathbb{R}$ lies in Conv $(W \mu)$ if and only if $\left\langle x, \widetilde{\omega}_{i}\right\rangle \leq\left\langle\mu, \widetilde{\omega}_{i}\right\rangle, \forall i \in I$, where, for any $i \in I, \widetilde{\omega}_{i}$ stands for the fundamental coweight corresponding to $\alpha_{i}$. One of the difficulties we encounter in proving $z^{\prime} \in \operatorname{Conv}(W \mu)$ is that the element $z^{\prime}$, like $z$, is not dominant in general. So, we let $w^{\prime} \in W$ be such that $w^{\prime}\left(z^{\prime}\right)$ is dominant. Then to show that $z^{\prime} \in \operatorname{Conv}(W \mu)$, it suffices to prove that $\left\langle w^{\prime}\left(z^{\prime}\right), \widetilde{\omega}_{i}\right\rangle \leq\left\langle\mu, \widetilde{\omega}_{i}\right\rangle, \forall i \in I$.

The strategy of the proof of the last inequalities consists on shifting the difficulty from proving these inequalities directly to constructing an element $w^{\prime}$ as above in such a way that we get the desired inequalities almost for free. To explain the strategy, let us first introduce some more terminology. For $\lambda \in P(R)$ and $w \in W$, we say that $w$ is $\lambda$-minuscule if there is a reduced expression $w=s_{i_{1}} s_{i_{2}} \cdots s_{i_{t}}$ such that

$$
s_{i_{r}} s_{i_{r+1}} \cdots s_{i_{t}} \lambda=\lambda+\alpha_{i_{r}}+\alpha_{i_{r+1}}+\cdots+\alpha_{i_{t}}, 1 \leq r \leq t,
$$

where, for any $i, s_{i} \in W$ stands for the simple reflection corresponding to $\alpha_{i}$. It is easily seen that $w$ is $\lambda$-minuscule if and only if

$$
\left\langle s_{i_{r+1}} \cdots s_{i_{t}} \lambda, \alpha_{i_{r}}^{\vee}\right\rangle=-1,1 \leq r \leq t .
$$

Note that, usually, one defines $\lambda$-minuscule Weyl group elements by requiring, in the last equalities, that the left-hand side is equal to +1 as opposed to -1 . For more on minuscule Weyl group elements, a notion invented by Peterson, see for example [22]. We point out that the notion of a Weyl group element $w$ being $\lambda$-minuscule does not depend on the choice of the reduced expression for $w$ (see [22, Proposition 2.1] for a proof).

The next result reveals what kind of $w^{\prime} \in W$ we are looking for and the reason for that.

Proposition 2.3. - Let $C_{\mu}^{+}:=\left\{x \in P(R) \otimes_{\mathbb{Z}} \mathbb{R} \mid\left\langle x, \widetilde{\omega}_{i}\right\rangle \leq\left\langle\mu, \widetilde{\omega}_{i}\right\rangle, \forall i \in I\right\}$ and suppose that $u \in C_{\mu}^{+}$. Then $w(u)$ lies in $C_{\mu}^{+}$for all $w \in W$ such that $w$ is u-minuscule.

We prove the proposition below, but first note that $z^{\prime} \in C_{\mu}^{+}$. Indeed, recall that $z^{\prime}=y+\sum_{j \in J} k_{j}^{\prime} \alpha_{j}$. All the numbers $k_{i}^{\prime}$ belong to the half-open interval $[0,1)$. We would like to prove that

$$
\left\langle z^{\prime}, \widetilde{\omega}_{i}\right\rangle \leq\left\langle\mu, \widetilde{\omega}_{i}\right\rangle, \forall i \in I \text {. }
$$

If $i \in I \backslash J$, then $\left\langle z^{\prime}, \widetilde{\omega}_{i}\right\rangle=\left\langle y, \widetilde{\omega}_{i}\right\rangle$. But, since $\operatorname{Conv}(W \mu) \subset C_{\mu}^{+}$and $y \in \operatorname{Conv}(W \mu)$, we have $\left\langle y, \widetilde{\omega}_{i}\right\rangle \leq\left\langle\mu, \widetilde{\omega}_{i}\right\rangle, \forall i \in I$. Therefore, $\left\langle z^{\prime}, \widetilde{\omega}_{i}\right\rangle \leq\left\langle\mu, \widetilde{\omega}_{i}\right\rangle, \forall i \in I \backslash J$.

If $i \in J$, then $\left\langle z^{\prime}, \widetilde{\omega}_{i}\right\rangle=\left\langle y, \widetilde{\omega}_{i}\right\rangle+k_{i}^{\prime}$. From $y \in \operatorname{Conv}(W \mu) \subset C_{\mu}^{+}$, we again have $\left\langle y, \widetilde{\omega}_{i}\right\rangle \leq\left\langle\mu, \widetilde{\omega}_{i}\right\rangle$. Moreover, since $y$ and $\mu$ have the same image in $P(R) / Q(R)$, we have $\left\langle\mu, \widetilde{\omega}_{i}\right\rangle-\left\langle y, \widetilde{\omega}_{i}\right\rangle \in \mathbb{Z}_{\geq 0}$. Using the facts that $k_{i}^{\prime} \in[0,1)$ and $\left\langle y, \widetilde{\omega}_{i}\right\rangle+k_{i}^{\prime} \in \mathbb{Z}$ we can then deduce that $\left\langle y, \widetilde{\omega}_{i}\right\rangle+k_{i}^{\prime} \leq\left\langle\mu, \widetilde{\omega}_{i}\right\rangle, \forall i \in J$, i.e., that $\left\langle z^{\prime}, \widetilde{\omega}_{i}\right\rangle \leq\left\langle\mu, \widetilde{\omega}_{i}\right\rangle, \forall i \in J$, and hence $z^{\prime} \in C_{\mu}^{+}$. 
REMARK 2.4. - The inequalities $\left\langle z^{\prime}, \widetilde{\omega}_{i}\right\rangle \leq\left\langle\mu, \widetilde{\omega}_{i}\right\rangle$ would not be trivial (or, rather, easily proved) if we had used $z$ instead of $z^{\prime}$ because the coefficients $k_{i}$ may be equal to or bigger than 1 . While these inequalities are satisfied for $z$, we do not know of a simple way to prove this fact, and can only deduce it after proving that $z$ lies in the Weyl orbit of $z^{\prime}$. In any case, for our proof, we do not need to show (directly) that $z$ satisfies these inequalities.

Using Proposition 2.3, we find that Proposition 2.2 follows if we show that there exists an element $w^{\prime} \in W$ such that $w^{\prime}\left(z^{\prime}\right)$ is dominant and $w^{\prime}$ is $z^{\prime}$-minuscule. Before we tackle this problem, let us prove Proposition 2.3.

Proof of Proposition 2.3. - Suppose that the conditions of the proposition are satisfied. Let $w \in W$ be $u$-minuscule and suppose that a reduced expression for $w$ is given by $s_{i_{1}} s_{i_{2}} \cdots s_{i_{t}}$. We use induction on $t$, the length of $w$, to prove that $w(u)$ lies in the cone $C_{\mu}^{+}$, with the case $t=0$ (i.e., $w=i d$ ) being assumed. Suppose that $s_{i_{r+1}} \cdots s_{i_{t}}(u)$ lies in $C_{\mu}^{+}$. We would like to prove that the element $s_{i_{r}} s_{i_{r+1}} \cdots s_{i_{t}}(u)$ also lies in $C_{\mu}^{+}$. Since $\left\langle s_{i_{r+1}} \cdots s_{i_{t}}(u), \alpha_{i_{r}}\right\rangle=-1$, we apply the simple reflection $s_{i_{r}}$ to $s_{i_{r+1}} \cdots s_{i_{t}}(u)$ to get $s_{i_{r}} s_{i_{r+1}} \cdots s_{i_{t}}(u)=s_{i_{r+1}} \cdots s_{i_{t}}(u)+\alpha_{i_{r}}$. Then, clearly, for any $i \in I \backslash\left\{i_{r}\right\}$, we have $\left\langle s_{i_{r}} s_{i_{r+1}} \cdots s_{i_{t}}(u), \widetilde{\omega}_{i}\right\rangle=\left\langle s_{i_{r+1}} \cdots s_{i_{t}}(u), \widetilde{\omega}_{i}\right\rangle \leq\left\langle\mu, \widetilde{\omega}_{i}\right\rangle$. For $i=i_{r}$ we have that $\left\langle s_{i_{r}} s_{i_{r+1}} \cdots s_{i_{t}}(u), \widetilde{\omega}_{i_{r}}\right\rangle=\left\langle s_{i_{r+1}} \cdots s_{i_{t}}(u), \widetilde{\omega}_{i_{r}}\right\rangle+1$. Since $\left\langle s_{i_{r+1}} \cdots s_{i_{t}}(u), \widetilde{\omega}_{i_{r}}\right\rangle \leq\left\langle\mu, \widetilde{\omega}_{i_{r}}\right\rangle$, it remains to show that we cannot have $\left\langle s_{i_{r+1}} \cdots s_{i_{t}}(u), \widetilde{\omega}_{i_{r}}\right\rangle=\left\langle\mu, \widetilde{\omega}_{i_{r}}\right\rangle$.

For a contradiction, suppose that $\left\langle s_{i_{r+1}} \cdots s_{i_{t}}(u), \widetilde{\omega}_{i_{r}}\right\rangle=\left\langle\mu, \widetilde{\omega}_{i_{r}}\right\rangle$. Then, since $s_{i_{r+1}} \cdots s_{i_{t}}(u) \in C_{\mu}^{+}$and $\mu$ is dominant, there exist non-negative reals $a_{i}, i \in I \backslash\left\{i_{r}\right\}$, so that

$$
s_{i_{r+1}} \cdots s_{i_{t}}(u)=\mu-\sum_{i \in I \backslash\left\{i_{r}\right\}} a_{i} \alpha_{i},
$$

and this contradicts our assumption that $\left\langle s_{i_{r+1}} \cdots s_{i_{t}}(u), \alpha_{i_{r}}^{\vee}\right\rangle=-1$, because $\left\langle\mu, \alpha_{i_{r}}^{\vee}\right\rangle \geq 0$, $a_{i}$ 's are non-negative, and $\left\langle\alpha_{i}, \alpha_{i_{r}}^{\vee}\right\rangle \leq 0, \forall i \neq i_{r}$.

Recall that we have reduced the proof of Proposition 2.2 to showing that there exists an element $w^{\prime} \in W$ such that $w^{\prime}\left(z^{\prime}\right)$ is dominant, and $w^{\prime}$ is $z^{\prime}$-minuscule. Initially, this problem was proved by the author on a case-by-case basis, but the following result from [6] greatly simplifies the proof.

Proposition $2.5([6])$. - Let $\lambda \in P(R)$. Then there exists an element $w \in W$ such that $w(\lambda)$ is dominant and $w$ is $\lambda$-minuscule if and only if

$$
\left\langle\lambda, \alpha^{\vee}\right\rangle \geq-1
$$

for all positive roots $\alpha$ of $R$.

In fact, in [6] a much more general result than Proposition 2.5 is proved, but we will only need the special case above. The proof of the proposition in this case is fairly elementary (but, for more details, see [6]). Here we include the proof of the only part of the proposition that we use: that (5) is a sufficient condition for the existence of $w$ as in the proposition. Let $\lambda \in P(R)$ satisfy (5), and consider the set

$$
\phi_{\lambda}:=\left\{\alpha \in R^{+}:\left\langle\lambda, \alpha^{\vee}\right\rangle<0\right\},
$$

$4^{\text {e }}$ SÉRIE - TOME $43-2010-\mathrm{N}^{\mathrm{o}} 6$ 
where $R^{+}$stands for the positive roots of $R$. Note that if $\left\langle\lambda, \alpha_{i}^{\vee}\right\rangle=-1$, then we have a natural bijection

$$
\phi_{\lambda} \backslash\left\{\alpha_{i}\right\} \longrightarrow \phi_{s_{i}(\lambda)}\left(\alpha \longmapsto s_{i}(\alpha)\right)
$$

So, when applying a $\lambda$-minuscule element $w \in W$ to $\lambda$, we get that the size of the set $\phi_{\lambda}$ decreases (by an element, for each simple reflection on the reduced expression for $w$ ). Clearly, this set is finite, therefore we see that there exists an element $w \in W$ such that $w$ is $\lambda$ minuscule and $w(\lambda)$ is dominant.

Using Proposition 2.5, Proposition 2.2 now follows from the next result.

Proposition 2.6. - For the element $z^{\prime}$ we have that $\left\langle z^{\prime}, \alpha^{\vee}\right\rangle \geq-1$, for all roots $\alpha \in R^{+}$.

The proof of the last result is carried out in the next section. In the end, let us mention that one can also phrase Propositions 2.5 and 2.6, as well as the results of the next section, using a modified version of the numbers game of Mozes (cf. [16]), where we impose a lower bound condition (see [6] for more details).

\section{Proof of Proposition 2.6}

The results in this section are joint with Travis Schedler (stemming from [6]). We work under the same assumptions as in the last section. In particular, $R$ is simply-laced. First, we prove that $z$ can be obtained from $z^{\prime}$ by applying a $z^{\prime}$-minuscule Weyl group element to $z^{\prime}$. More generally, we have:

Proposition 3.1. - Suppose $u \in P(R)$ is minuscule, and

$$
u=\sum_{i \in I} \ell_{i} \alpha_{i}
$$

with $\ell_{i} \geq 0$ for all $i$. Let $u^{\prime}$ be the fractional part of $u$, given by

$$
u^{\prime}=\sum_{i \in I} \ell_{i}^{\prime} \alpha_{i}, \quad \ell_{i}^{\prime}=\ell_{i}-\left\lfloor\ell_{i}\right\rfloor .
$$

Then there exists an element $w \in W$ such that $w$ is $u^{\prime}$-minuscule and $w\left(u^{\prime}\right)=u$.

A corollary of this proposition is that, if $u \in P(R)$ is minuscule, then $u^{\prime}$ is in the Weyl orbit of $u$, and thus, using [3, Ch. VIII, §7, Prop. 6], we can conclude that $u^{\prime}$ is itself minuscule. In particular, since the element $z$ from the previous section is $J$-minuscule, the proposition shows that its $J$-fractional part $z^{\prime}$ is also $J$-minuscule. We give a non-case-by-case proof of the above proposition in Subsection 3.2 below. (The only classification result used is the fact that all simply-laced Dynkin diagrams are star-shaped graphs.) First, we continue with the proof of Proposition 2.6, which will be deduced from the next result.

Proposition 3.2. - Let $u \in Q\left(R_{J}\right) \otimes_{\mathbb{Z}} \mathbb{R}$ be an element such that $\left\langle u, \alpha^{\vee}\right\rangle \in[-1,1]$ for all $\alpha \in R_{J}$. Then, $\left\langle u, \beta^{\vee}\right\rangle \in(-2,2)$ for all $\beta \in R$. 
Proof of Proposition 2.6. Recall that we would like to prove that $\left\langle z^{\prime}, \alpha^{\vee}\right\rangle \geq-1$, for all $\alpha \in R^{+}$, where $R^{+}$is the set of positive roots of $R$. Put $u:=z^{\prime}-y$ and note that $u$ satisfies the conditions of the proposition above: Indeed, as remarked above, due to Proposition 3.1, we have that $z^{\prime}$ is $J$-minuscule. Also, $y$ is orthogonal to all the coroots of $R_{J}$ (recall the remarks after the short exact sequence (4) in the previous section). So, we have $u \in Q\left(R_{J}\right) \otimes_{\mathbb{Z}} \mathbb{R}$ and $\left\langle u, \alpha^{\vee}\right\rangle \in[-1,1], \forall \alpha \in R_{J}$.

Therefore Proposition 3.2 implies, in particular, that $\left\langle u, \alpha^{\vee}\right\rangle>-2$ for all $\alpha \in R^{+}$. Since $y$ is dominant and $\left\langle z^{\prime}, \alpha^{\vee}\right\rangle \in \mathbb{Z}$, we then obtain

$$
\left\langle z^{\prime}, \alpha^{\vee}\right\rangle=\left\langle u, \alpha^{\vee}\right\rangle+\left\langle y, \alpha^{\vee}\right\rangle \geq-1, \forall \alpha \in R^{+},
$$

as desired.

3.1. - In this subsection we prove Proposition 3.2.

Proof of Proposition 3.2. - Let us denote

$$
\mathcal{M}_{J}:=\left\{v \in Q\left(R_{J}\right) \otimes_{\mathbb{Z}} \mathbb{R} \mid\left\langle v, \alpha^{\vee}\right\rangle \in[-1,1], \forall \alpha \in R_{J}\right\} .
$$

Since $R=R^{+} \sqcup\left(-R^{+}\right)$, it suffices to show that if $\beta \in R^{+} \backslash R_{J}$, then $\left\langle u, \beta^{\vee}\right\rangle>-2$ for all $u \in M_{J}$.

Fix once and for all (in this subsection) an element $\beta \in R^{+} \backslash R_{J}$. Then we have

Claim 3.3. - The minimum value of the function $\Upsilon: \mathcal{M}_{J} \longrightarrow \mathbb{R}, u \longmapsto\left\langle u, \beta^{\vee}\right\rangle$, is attained when $u=(-\beta)_{J}$, the projection of $-\beta$ to $Q\left(R_{J}\right) \otimes_{\mathbb{Z}} \mathbb{R}$ with respect to the Cartan form.

Since $\beta \in R \backslash R_{J}$, we have $-\beta \notin Q\left(R_{J}\right) \otimes_{\mathbb{Z}} \mathbb{R}$, and it follows that $\left\langle(-\beta)_{J}, \beta^{\vee}\right\rangle>\left\langle\beta,-\beta^{\vee}\right\rangle=-2$. Thus, assuming Claim 3.3 is true, we have that $\Upsilon(u)>-2, \forall u \in \mathcal{M}_{J}$, proving Proposition 3.2. The rest of this subsection deals with the proof of Claim 3.3.

Claim 3.4. - We may assume that $\left\langle\alpha_{j}, \beta^{\vee}\right\rangle \leq 0$, for all $j \in J$.

Proof. - First, if there exists an element $j \in J$ such that $\left\langle\alpha_{j}, \beta^{\vee}\right\rangle>0$, then $\left\langle\alpha_{j}, \beta^{\vee}\right\rangle=1$ (since we are working with simply-laced root systems, we necessarily have $\left\langle\alpha_{j}, \beta^{\vee}\right\rangle \leq 1$ ). Second, we may replace $\beta$ with $s_{j} \beta$ and apply the automorphism $s_{j}$ to $\mathcal{M}_{J}$, without changing the statement of Claim 3.3. Since by applying a series of simple reflections to $\beta$, we can ensure that the obtained element is anti-dominant, we may therefore assume that $\left\langle\alpha_{j}, \beta^{\vee}\right\rangle \leq 0$, for all $j \in J$.

Let us identify the subsets of $I$ with the subgraphs of the Dynkin diagram $\Gamma_{I}$ of $R$. For example, by connected components of the subset $J \subset I$ we mean the connected components of the subgraph $\Gamma_{J} \subset \Gamma_{I}$ that corresponds to $J$. Denote by $J_{1}, \ldots, J_{m}$ the connected components of $J$.

Claim 3.5. - For each connected component $J_{p}$, there exists at most one element in $J_{p}$, denoted by $j_{p}$, such that $\left\langle\alpha_{j_{p}}, \beta^{\vee}\right\rangle<0$. Moreover, whenever this element exists, we have $\left\langle\alpha_{j_{p}}, \beta^{\vee}\right\rangle=-1$. 
Proof. - The second statement is clear, since we are working with simply-laced root systems. For the first one, assume that $\left\langle\alpha_{j_{p}}, \beta^{\vee}\right\rangle<0$ and $\left\langle\alpha_{j_{p}^{\prime}}, \beta^{\vee}\right\rangle<0$, for two elements $j_{p}, j_{p}^{\prime} \in J_{p}$, and consider the root that is the sum of the simple roots $\alpha_{i}$, where $i$ ranges through the set of vertices that, in the Dynkin diagram $\Gamma_{I}$, form a line segment that starts at $j_{p}$ and ends at $j_{p}^{\prime}$, and that is entirely contained in $J_{p}$. Then pairing $\beta^{\vee}$ with that root gives at most -2 , a contradiction with the fact that we are working with a simply-laced root system.

We may assume that there exists a $j_{p}$ as in the last claim in each connected component of $J$, since otherwise we could delete the whole connected component from $J$ without changing the statement of the Claim 3.3.

Next, note that by assumption we have $(-\beta)_{J} \in \mathcal{M}_{J}$, and thus the function $\Upsilon: \mathcal{M}_{J} \longrightarrow \mathbb{R}$ attains its minimum value at $(-\beta)_{J}$ if and only if for every element $\gamma \in Q\left(R_{J}\right) \otimes_{\mathbb{Z}} \mathbb{R}$ such that $\left\langle\gamma, \beta^{\vee}\right\rangle<0$, and for every $t>0$, we have that $(-\beta)_{J}+t \gamma \notin \mathcal{M}_{J}$.

Suppose that $\gamma=\sum_{j \in J} c_{j} \alpha_{j} \in Q\left(R_{J}\right) \otimes_{\mathbb{Z}} \mathbb{R}$ satisfies $\left\langle\gamma, \beta^{\vee}\right\rangle<0$. Then we have $\sum_{p=1}^{m} c_{j_{p}}>0$. Now, pick $p$ such that $c_{j_{p}}>0$. Let $J_{p}^{\prime} \subset J_{p}$ be the maximal connected subset such that $j_{p} \in J_{p}^{\prime}$ and $c_{j}>0$ for all $j \in J_{p}^{\prime}$. Let $\alpha^{\vee} \in\left(R_{J_{p}^{\prime}}\right)_{+}$be the maximal coroot of $J_{p}^{\prime}$. Then we have $\left\langle\beta, \alpha^{\vee}\right\rangle=-1$, so $\left\langle(-\beta)_{J}, \alpha^{\vee}\right\rangle=1$. Also, we have

$$
\begin{gathered}
\left\langle\alpha_{j}, \alpha^{\vee}\right\rangle \geq 0, \forall j \in J_{p}^{\prime} \text { (with strict inequality for at least one } j \text { ), } \\
\qquad\left\langle\alpha_{j}, \alpha^{\vee}\right\rangle \leq 0, \forall j \notin J_{p}^{\prime}, \\
c_{j}>0, \forall j \in J_{p}^{\prime} \text {, and } c_{j} \leq 0, \forall j \notin J_{p}^{\prime} \text { adjacent to } J_{p} .
\end{gathered}
$$

Thus, we deduce that

$$
\left\langle\gamma, \alpha^{\vee}\right\rangle=\sum_{j \in J_{p}^{\prime}} c_{j}\left\langle\alpha_{j}, \alpha^{\vee}\right\rangle+\sum_{\substack{j \in J_{p} \backslash J_{p}^{\prime} \\ j \text { adjacent to } J_{p}^{\prime}}} c_{j}\left\langle\alpha_{j}, \alpha^{\vee}\right\rangle>0 .
$$

We therefore get $\left\langle(-\beta)_{J}+t \gamma, \alpha^{\vee}\right\rangle=1+t\left(\left\langle\gamma, \alpha^{\vee}\right\rangle\right)>1$ for all $t>0$, and hence $(-\beta)_{J}+t \gamma \notin \mathcal{M}_{J}$ for any $t>0$. Hence, the function $\Upsilon: \mathcal{M}_{J} \longrightarrow \mathbb{R}$ indeed attains its minimum value at $(-\beta)_{J}$, proving Claim 3.3.

\section{2. - Next we prove Proposition 3.1.}

Proof of Proposition 3.1. - We will use two expressions for $u$ : One, $u=\sum_{i \in I} \ell_{i} \alpha_{i}$, in terms of simple roots $\alpha_{i}, i \in I$; the other, $u=\sum_{i \in I} u_{i} \omega_{i}$, in terms of fundamental weights $\omega_{i}, i \in I$, where $\omega_{i}$ corresponds to $\alpha_{i}^{\vee}$. Note that $u_{i}=\left\langle u, \alpha_{i}^{\vee}\right\rangle$ and $\ell_{i}=\left\langle u, \widetilde{\omega}_{i}\right\rangle$, where we recall that $\widetilde{\omega}_{i}$ is the fundamental coweight corresponding to $\alpha_{i}$. Also, for every $c \in I$, we have $u_{c}=2 \ell_{c}-\sum_{i m c} \ell_{i}$, where $i \leadsto c$ means that the vertices $i$ and $c$ of the Dynkin diagram $\Gamma_{I}$ are adjacent to each other. Finally, note that since $u$ is minuscule, we have $u_{i} \in\{-1,0,1\}, \forall i \in I$.

Our proposition follows from

Claim 3.6. - There exists an element $i \in I$ such that $u_{i}=1$ and $\ell_{i} \geq 1$. 
Indeed, by using induction on $\sum_{i \in I}\left\lfloor\ell_{i}\right\rfloor$, the claim allows us to apply the simple reflection $s_{i}$ to $u$ and we can then use the induction hypothesis, for the modified $u$ and the same $u^{\prime}$, to get the desired result. (We remark that the base case of the induction, when $\sum_{i \in I}\left\lfloor\ell_{i}\right\rfloor=0$, corresponds to $u=u^{\prime}$, in which case Proposition 3.1 is trivially true.)

We may and will assume henceforth that $u \neq u^{\prime}$. The rest of this subsection is devoted to the proof of Claim 3.6. For a contradiction, assume that

(b) There does not exist an $i \in I$ such that $u_{i}=1$ and $\ell_{i} \geq 1$.

We will need a number of results to prove that the assumption (b) leads to a contradiction.

Claim 3.7. - There exists an element $i_{0} \in I$ such that $\ell_{i_{0}} \geq \ell_{i}, \forall i \in I$, and such that $\ell_{i_{0}}>\ell_{i}$ for some $i$ that is adjacent to $i_{0}$ in the Dynkin diagram $\Gamma_{I}$ of $R$. Moreover, we must have $\ell_{i_{0}} \geq 1$.

Proof. - Suppose, for a contradiction, that all $\ell_{i}$ 's are equal to each other. If the graph $\Gamma_{I}$ has only one vertex, then we get $u=u^{\prime}$, contradicting our assumption that $u \neq u^{\prime}$.

Now suppose that $\Gamma_{I}$ has more than one vertex. Let $d_{1}$ be a vertex in $\Gamma_{I}$ of valence 1 and let $d_{2}$ be the unique vertex in $\Gamma_{I}$ such that $d_{2}$ is adjacent to $d_{1}$. We have $u_{d_{1}}=\left\langle u, \alpha_{d_{1}}^{\vee}\right\rangle=\left\langle\sum_{i \in I} \ell_{i} \alpha_{i}, \alpha_{d_{1}}^{\vee}\right\rangle=2 \ell_{d_{1}}-\ell_{d_{2}}$. Since we assumed that all $\ell_{i}$ 's are equal to each other, we then get that $\ell_{i}=\ell_{d_{1}}=u_{d_{1}}, \forall i \in I$. Also, since $u$ is minuscule (or more precisely since $u_{d_{1}} \in\{-1,0,1\}$ ) and $\ell_{i} \geq 0$, we get that $\ell_{i}$ 's are either all equal to zero or all equal to one: In the former case we get that $u=u^{\prime}$, a contradiction; in the latter case we get that $\left\langle u, \delta^{\vee}\right\rangle \geq 2$, where $\delta^{\vee}$ is the maximal positive coroot in $R$, contradicting the minusculity of $u$. In any case, we get a contradiction and the first assertion of the claim follows. The inequality $\ell_{i_{0}} \geq 1$ follows from the assumption $u \neq u^{\prime}$.

Claim 3.8. - The vertex $i_{0} \in I$, from the previous claim, has valence 3 in the Dynkin $\operatorname{graph} \Gamma_{I}$.

Proof. - Since $\ell_{i_{0}} \geq 1$, the assumption (b) yields $u_{i_{0}} \in\{0,-1\}$. If $i_{0}$ had valence $\leq 2$, then $u_{i_{0}}=2 \ell_{i_{0}}-\sum_{i m \rightarrow i_{0}} \ell_{i}$ would give $u_{i_{0}}>0$, a contradiction. Thus, the valence of $i_{0}$ is 3 , and $i_{0}$ is the node of $\Gamma_{I}$.

Since $R$ is a simply-laced root system, as a corollary of the last claim we get that $\Gamma_{I}$ is a Dynkin diagram of type $D$ or $E$, but not $A$. We will think of $\Gamma_{I}$ as a star with three branches, denoted by $\Gamma_{1}, \Gamma_{2}$ and $\Gamma_{3}$, each of which contains the node $i_{0}$. Let us denote the vertices of $\Gamma_{1}$ and $\Gamma_{2}$ and $\Gamma_{3}$, from the node to the respective vertex of valence one, by $i_{0}, i_{1}, \ldots, i_{a_{1}}$, and $i_{0}, i_{a_{1}+1}, \ldots, i_{a_{2}}$, and $i_{0}, i_{a_{2}+1}, \ldots, i_{a_{3}}$, respectively. Note that the vertices of $\Gamma_{I}$ are then $i_{0}, \ldots, i_{a_{3}}$, where $0<a_{1}<a_{2}<a_{3}$ are integers.

Let $\Gamma^{\prime} \subset \Gamma_{I}$, on the vertex set $I^{\prime} \subset I$, be the maximal connected subgraph containing $i_{0}$ such that $u_{i} \in\{0,-1\}$ for all $i \in I^{\prime}$. Let $\mathcal{T}:=\left\{t \in I \backslash I^{\prime} \mid t\right.$ is adjacent to $\left.\Gamma^{\prime}\right\}$. For every $t \in \mathcal{T}$, we have $u_{t}=1$.

Claim 3.9. - The set $\mathcal{T}$ is non-empty.

Proof. - If this were not the case, we would have $u_{i} \leq 0, \forall i \in I$. Since $u$ is minuscule and $u \neq 0$ (the latter follows from $u \neq u^{\prime}$ ), we get that $u=-\omega_{c}$ for some $c \in I$, contradicting the assumption that $u=\sum_{i \in I} \ell_{i} \alpha_{i}$, where $\ell_{i} \geq 0, \forall i$. The assertion of the claim follows. 
Claim 3.10. - There exists one and only one $i_{p} \in I^{\prime}$ such that $u_{i_{p}} \neq 0$.

Proof. - Since $u$ is minuscule, we have that at most one $u_{i}, i \in I^{\prime}$, is non-zero, and the restriction $\left.u\right|_{\Gamma^{\prime}}$ is anti-dominant and minuscule on $\Gamma^{\prime}$. Suppose, for a contradiction, that $u_{i}=0, \forall i \in I^{\prime}$. Then, from Claim 3.9, we deduce that $u=\omega_{i_{t}}$, for some $t \in\left[0, a_{3}\right] \cap \mathbb{Z}$. Assume, without loss of generality, that $t \in\left(0, a_{1}\right]$, i.e., $i_{t}$ belongs to the branch $\Gamma_{1}$, but is not the node $i_{0}$. We will contradict the assumption (b) by proving that $\ell_{i_{t}} \geq 1$ : First, note that we have $u_{i_{c}}=2 \ell_{i_{c}}-\ell_{i_{c-1}}-\ell_{i_{c+1}}, \forall c \in[1, t] \cap \mathbb{Z}$ (where, if $t=a_{1}$, we disregard the term $\left.\ell_{i_{t+1}}\right)$. Using $\sum_{b=1}^{t} u_{i_{b}}=1$ we therefore get $-\ell_{i_{0}}+\ell_{i_{1}}+\ell_{i_{t}}-\ell_{i_{t+1}}=1$. From $\ell_{i_{0}}-\ell_{i_{1}} \geq 0$ and $\ell_{i_{t+1}} \geq 0$ we conclude $\ell_{i_{t}}=1+\ell_{i_{t+1}}+\left(\ell_{i_{0}}-\ell_{i_{1}}\right) \geq 1$, which provides the desired contradiction and proves our claim.

The next result studies the possibilities for the number of the connected components of $\Gamma_{I} \backslash \Gamma^{\prime}$.

Claim 3.11. - The graph $\Gamma_{I} \backslash \Gamma^{\prime}$ has exactly two connected components.

Proof. - That $\Gamma_{I} \backslash \Gamma^{\prime}$ has at least one connected component is guaranteed by Claim 3.9. Obviously, $\Gamma_{I} \backslash \Gamma^{\prime}$ has at most three connected components. Suppose, for a contradiction, that it has three connected components. Let $t_{1}, t_{2}, t_{3}$ be three distinct elements of $\mathcal{T}$. Then the coroot $\alpha^{\vee}$ that is the sum of all the simple coroots $\alpha_{i}^{\vee}$, with $i$ ranging through the vertices of $\Gamma^{\prime} \cup\left\{t_{1}, t_{2}, t_{3}\right\}$, is such that $\left\langle u, \alpha^{\vee}\right\rangle>1$, contradicting the minusculity of $u$. Thus we conclude that $\Gamma_{I} \backslash \Gamma^{\prime}$ has at least one and at most two connected components.

It remains to prove that $\Gamma_{I} \backslash \Gamma^{\prime}$ cannot have a unique connected component. Assume, for a contradiction, that $\Gamma_{I} \backslash \Gamma^{\prime}$ has a unique connected component. Then the set $\mathcal{T}$ is a singleton, so we let $\mathcal{T}=\left\{i_{t}\right\}$. Let $i_{p}$ be the vertex of $\Gamma^{\prime}$ such that $u_{i_{p}}=-1$ (see Claim 3.10). We will distinguish two cases: $(a)$ the vertex $i_{p}$ is adjacent to $i_{t}$; and $(b)$ the vertex $i_{p}$ is not adjacent to $i_{t}$.

Case (a): Suppose that the vertex $i_{p}$ is adjacent to $i_{t}$. This implies $p=t-1$. Without loss of generality, assume that the vertex $i_{t}$ lies in the branch $\Gamma_{1}$ of $\Gamma_{I}$, i.e., $t \in\left(0, a_{1}\right] \cap \mathbb{Z}$.

Since $u$ is minuscule, it remains so when restricted to the line segment $\left[i_{t-1}, i_{a_{1}}\right] \cap \mathbb{Z}$ of $\Gamma_{I}$. In addition, $u$ is zero in the complement of that segment, i.e., $u_{i}=0$ for all $i$ that lie outside $\left[i_{t-1}, i_{a_{1}}\right] \cap \mathbb{Z}$. It is clear that there exists an element $w \in W$ such that $w$ is $u$-minuscule and $w(u)=-\omega_{i_{q}}$, for some $q \in\left[t, a_{1}\right] \cap \mathbb{Z}$, where the simple reflections $s_{i_{b}}$ appearing in a reduced expression of $w$ are such that $b \in\left[t, a_{1}\right] \cap \mathbb{Z}$. We therefore have that $w(u)=u-\sum_{b=t}^{a_{1}} d_{b} \alpha_{i_{b}}$, where $d_{b} \in \mathbb{Z}_{\geq 0}, \forall b$. Since $u=\sum_{b=0}^{a_{3}} \ell_{i_{b}} \alpha_{i_{b}}$ and $w(u)=-\omega_{i_{q}}=-\sum_{b=0}^{a_{3}} e_{b} \alpha_{i_{b}}$, where $e_{b} \in \mathbb{Z}_{\geq 0}, \forall b$, we deduce that $\sum_{b=0}^{a_{3}} e_{b} \alpha_{i_{b}}+\sum_{b=0}^{a_{3}} \ell_{i_{b}} \alpha_{i_{b}}=\sum_{b=t}^{a_{1}} d_{b} \alpha_{i_{b}}$. In the last identity, the coefficient in front of $\alpha_{i_{0}}$ is $e_{i_{0}}+\ell_{i_{0}}>0$ on the left hand side and 0 on the right hand side, contradicting the linear independence of the elements of $\left\{\alpha_{i_{b}}\right\}_{b=0}^{a_{3}}$.

Case (b): Suppose now that the vertex $i_{p}$ is not adjacent to $i_{t}$. We may assume that $i_{p}$ lies in the branch $\Gamma_{1}$. Consider the element $v:=s_{i_{0}} \cdots s_{i_{p-1}} s_{i_{p}}(u)$, and note that it is minuscule, since it lies in the Weyl orbit of $u$. However, we also have that $\left\langle v, \alpha^{\vee}\right\rangle=2$, where $\alpha^{\vee}$ is the coroot that is the sum of the simple coroots corresponding to the node $i_{0}$ and its three adjacent vertices $i_{1}, i_{a_{1}+1}$ and $i_{a_{2}+1}$. Thus, we get a contradiction, proving that $i_{p}$ is not adjacent to $i_{t}$. Together with the case $(a)$ this implies that $\Gamma_{I} \backslash \Gamma^{\prime}$ cannot have only one connected component. The assertion of the claim follows. 
Now that we know that $\Gamma_{I} \backslash \Gamma^{\prime}$ has exactly two connected components, we may assume, without loss of generality, that these components lie in $\Gamma_{1} \cup \Gamma_{2}$. Let $\mathcal{T}=\left\{i_{t_{1}}, i_{t_{2}}\right\}$, where $t_{1} \in\left(0, a_{1}\right] \cap \mathbb{Z}$ and $t_{2} \in\left(a_{1}, a_{2}\right] \cap \mathbb{Z}$.

Claim 3.12. - The vertex $i_{p} \in \Gamma^{\prime}$, from Claim 3.10, lies in $\Gamma_{1} \cup \Gamma_{2}$.

Proof. - If $i_{p}$ lies outside $\Gamma_{1} \cup \Gamma_{2}$, then consider the coroot $\alpha^{\vee}$, which is the sum of the simple coroots $\alpha_{i}^{\vee}$ where $i$ ranges through the line subsegment of $\Gamma_{I}$ with endpoints the vertices $i_{t_{1}}$ and $i_{t_{2}}$ (on this segment, all the numbers $u_{i}$ are zero, except at the endpoints, where they are both 1 ). Then we get that $\left\langle u, \alpha^{\vee}\right\rangle=2$, contradicting the minusculity of $u$.

Using the last claim, and without loss of generality, assume that $i_{p} \in \Gamma_{2}$. We distinguish two cases: (i) The vertex $i_{t_{1}}$ is not adjacent to the node, and (ii) The vertex $i_{t_{1}}$ is adjacent to the node.

Case (i): Assume that the vertex $i_{t_{1}}$ is not adjacent to the node $i_{0}$. As in Claim 3.10, using $u_{i_{b}}=2 \ell_{i_{b}}-\sum_{i_{c} \rightsquigarrow i_{b}} \ell_{i_{c}}$, and the fact that $\sum_{b=1}^{t_{1}} u_{i_{b}}=1$, we get $-\ell_{i_{0}}+\ell_{i_{1}}+\ell_{i_{t_{1}}}-\ell_{i_{t_{1}+1}}=1$, or equivalently $\ell_{i_{t_{1}}}=1+\ell_{i_{t_{1}+1}}+\left(\ell_{i_{0}}-\ell_{i_{1}}\right)$ (where, if $t_{1}=a_{1}$, we disregard the term $\ell_{i_{t_{1}+1}}$ ). Since $\ell_{i_{0}} \geq \ell_{i_{1}}$, we deduce that $\ell_{i_{1}} \geq 1$, contradicting the assumption (b)

Case (ii): Assume now that the vertex $i_{t_{1}}$ is adjacent to the node $i_{0}$, i.e. $t_{1}=1$. Then, since $u_{1}=1$, we have that $2 \ell_{i_{1}}-\ell_{i_{0}}-\ell_{i_{2}}=1$, which implies $\ell_{i_{1}}=\frac{1+\ell_{i_{0}}+\ell_{i_{2}}}{2}$ (where, if $t_{1}=a_{1}=1$, we disregard the term $\ell_{i_{2}}$ ). Since $\ell_{i_{0}} \geq 1$ (see Claim 3.7), we deduce that $\ell_{i_{1}} \geq 1$, contradicting (b).

We have thus demonstrated that the assumption (b) results in a contradiction, and we can therefore conclude that Claim 3.6 holds. This also finishes the proof of Proposition 3.1.

\section{The non-simply laced cases}

In this section we will prove Theorem 2.1 for non-simply laced groups. We will use a folding argument to deduce the non-simply laced cases from the simply-laced ones. We thank Robert Kottwitz for generously sharing with us his ideas on proofs of the results in this section.

We retain the same notation as in the introduction. In particular, $G$ is a split connected reductive group, $B$ is a Borel subgroup, and $T$ is a maximal torus in $B$. We will, furthermore, suppose that $G$ is adjoint and simply-laced. Fix a set of root vectors $\left\{X_{\alpha}\right\}_{\alpha \in \Delta}$ of $T$, where $\Delta$ is the set of simple roots of $G$, with respect to the chosen Borel group $B$.

Let $\theta$ be an automorphism of $G$ that fixes $B, T$, and $\left\{X_{\alpha}\right\}_{\alpha \in \Delta}$, and such that the following holds:

(†) For every root $\alpha$ from $\Delta$, we have that $\alpha$ is orthogonal to every $\operatorname{root} \beta \neq \alpha$ that is in the orbit of $\alpha$ under the group generated by $\theta$, i.e., $(\alpha, \beta)=0$, for all $\beta \neq \alpha$ of the form $\beta=\theta^{k}(\alpha)$, for some $k \in \mathbb{N}$, 
where the parentheses $\left(\right.$, ) stand for the obvious bilinear pairing in $X^{*}(T) \otimes_{\mathbb{Z}} \mathbb{R}$.

Since $\theta$ acts on $T$, it also acts on the group of characters $X^{*}(T)$. Denote by $T^{\theta}$ the group of fixed points of $T$ under $\theta$. Then we have that

$$
X^{*}\left(T^{\theta}\right)=X^{*}(T)_{\theta},
$$

where $X^{*}(T)_{\theta}$ denotes the group of co-invariants of $X^{*}(T)$ under $\theta$. (In general, for an object on which the map $\theta$ acts, let us agree to use the superscript and subscript $\theta$ for the invariants and co-invariants, respectively, of this object under the action of $\theta$.)

It is clear that $\theta$ acts on $\Delta$. For each orbit of $\theta$ in $\Delta$ we pick a representative, giving us a set which we denote by $\mathscr{R}$ and which we assume is fixed for the rest of this section. The images in $X^{*}(T)_{\theta}$ of the elements of $\mathscr{R}$ give a basis for $X^{*}(T)_{\theta}$, and the latter is torsion-free. This means that $X^{*}\left(T^{\theta}\right)$ is torsion-free and hence $T^{\theta}$ is connected, which implies that $H:=G^{\theta}$ is also connected. Moreover, $H$ is adjoint since $G$ was assumed to be so. One gets all split adjoint $H$ (up to isomorphism) in this way. We remind the reader (cf. [3, Exercise VII, §5, 13, pp. 228-229]) that if the Dynkin diagram (or more generally an irreducible component thereof) corresponding to $G$ is of type $A_{2 n+1}(n \geq 1), D_{n}(n \geq 4), E_{6}$, or $D_{4}$, then the Dynkin diagram (or the respective irreducible component thereof) corresponding to $H$ is of type $B_{n}$, $C_{n-1}, F_{4}$, or $G_{2}$, respectively, where $\theta$ is of order two in each of the first three cases, apart from the last case where it is of order three.

Recall that by $X$ we have denoted the group of cocharacters $X_{*}(T)$. We write $Y$ for the group $X^{\theta}$ and note that in fact $Y=X_{*}\left(T^{\theta}\right)$. We now consider

$$
H \supset B^{\theta} \supset T^{\theta}
$$

and the Weyl group $W_{H}$ corresponding to $H$. Since $\operatorname{Cent}_{G}\left(T^{\theta}\right)=T$ (see [20, Ch. 10, p. 183, paragraph after Proposition 10.3.5]), we get $N_{G}\left(T^{\theta}\right) \subset N_{G}(T)$, and thus $W_{H} \leq W$.

In $H$, any Levi component $M_{H} \supset T^{\theta}$ of a parabolic subgroup containing $B^{\theta}$ arises as the fixed-points group $M^{\theta}$ for some $\theta$-stable Levi component $M \supset T$ of a parabolic subgroup (of $G$ ) containing $B$. We will write $M_{H}$ instead of $M^{\theta}$, and remark that it is connected, since $T^{\theta}$ is connected.

We need some more notation. We write $Y_{H}$ and $Y_{M_{H}}$ for the quotient of $Y$ by the coroot lattice for $H$ and $M_{H}$, respectively. The maps $\psi_{H}: Y \rightarrow Y_{H}$ and $\psi_{M_{H}}: Y \rightarrow Y_{M_{H}}$ are the natural projections. We write $\mathfrak{b}=Y \otimes_{\mathbb{Z}} \mathbb{R}$ and $\mathfrak{b}_{M_{H}}=Y_{M_{H}} \otimes_{\mathbb{Z}} \mathbb{R}$. The $\operatorname{map}_{M_{H}}: \mathfrak{b} \rightarrow \mathfrak{b}_{M_{H}}$ is the natural projection induced by $\psi_{M_{H}}$. Finally, for any coweight $\mu \in Y, \operatorname{Conv}\left(W_{H}(\mu)\right)$ stands for the convex hull in $\mathfrak{b}$ of all the weights in the orbit of $\mu$ under $W_{H}$.

Let $\mu \in Y$ be $H$-dominant. We define

$$
\mathscr{P}_{\mu, H}=\left\{\nu \in Y:(\mathrm{i}) \psi_{H}(\nu)=\psi_{H}(\mu) \text {; and (ii) } \nu \in \operatorname{Conv}\left(W_{H}(\nu)\right)\right\} \text {. }
$$

The following result implies Theorem 2.1 for non-simply laced adjoint groups. But, if Theorem 2.1 holds for the adjoint group of $G$, then it holds for $G$ itself (see [12, Fact 2, p. 167]). Therefore the result below implies Theorem 2.1 for all non-simply laced $G$, not just the adjoint ones. 
Proposition 4.1. - With notation as above, we have that

$$
\psi_{M_{H}}\left(\mathscr{P}_{\mu, H}\right)=\left\{\nu \in Y_{M_{H}} \text { :(i) } \nu, \mu \text { have the same image in } Y_{H}\right. \text {; }
$$

(ii) the image of $\nu$ in $\mathfrak{b}_{M_{H}}$ lies in $\left.\operatorname{pr}_{M_{H}}\left(\operatorname{Conv}\left(W_{H}(\mu)\right)\right)\right\}$.

Before we begin the proof of this proposition, we prove some useful results. First, a remark.

Remark 4.2. - Let us denote by $\theta_{\alpha}$ the orbit of $\alpha$ in $\Delta$ under $\theta$. Because of the condition $(\dagger)$ on $\theta$, we have that the coroots corresponding to the simple roots for $\left(T^{\theta}, H\right)$ are $N\left(\alpha^{\vee}\right):=\sum_{\gamma \in \vartheta_{\alpha}} \gamma^{\vee}$, where $\alpha$ varies through $\mathscr{R}$. We will need this fact in the proofs of the results that follow. The condition $(\dagger)$ guarantees that our answer is not $2 N\left(\alpha^{\vee}\right)$, which could otherwise happen for certain automorphisms $\theta$ (see [3, Ch. VIII, §5, Ex. 13, p. 13]).

Lemma 4.3. - Let $\mu \in Y$. Then $\mu$ is $H$-dominant if and only if $\mu$ is $G$-dominant.

Proof. - The statement of the lemma is a direct consequence of the fact that the simple roots for $\left(T^{\theta}, H\right)$ are restrictions to $T^{\theta}$ of the simple roots for $(T, G)$ and the condition that $\mu \in Y$.

Lemma 4.4. - Let $\mu, \nu \in Y$. Then $\nu \stackrel{G}{\leq} \mu \Longleftrightarrow \nu \stackrel{H}{\leq} \mu$.

Proof. - Recall that $\nu \stackrel{G}{\leq} \mu$, respectively $\nu \stackrel{H}{\leq} \mu$, means precisely that $\mu-\nu$ is a nonnegative integral linear combination of the coroots corresponding to the simple roots for $G$, respectively for $H$. We have that

$$
\nu \stackrel{G}{\leq} \mu \Longleftrightarrow \mu-\nu=\sum_{\alpha \in \Delta} c_{\alpha} \alpha^{\vee}
$$

for some $c_{\alpha} \in \mathbb{Z}_{\geq 0}$. Note that since $\mu$ and $\nu$ are fixed by $\theta$, the coefficients $c_{\alpha}$ are constant on the orbits of $\theta$ on the set of the simple roots from $\Delta$. Because of the equivalence $(\ddagger)$, we must have

$$
\nu \stackrel{G}{\leq} \mu \Longleftrightarrow \mu-\nu=\sum_{\alpha \in \mathscr{R}} d_{\alpha} N\left(\alpha^{\vee}\right),
$$

for some $d_{\alpha} \in \mathbb{Z}_{\geq 0}$. But, as mentioned in Remark 4.2, the coroots corresponding to the simple roots for $\left(T^{\theta}, H\right)$ are $N\left(\alpha^{\vee}\right)$, where $\alpha$ varies through $\mathscr{R}$. Hence, the last equivalence, according to the definition of $\stackrel{H}{\leq}$, yields

$$
\nu \stackrel{G}{\leq} \mu \Longleftrightarrow \nu \stackrel{H}{\leq} \mu,
$$

which we wanted to prove.

Lemma 4.5. - Let $\mu \in Y \subset X$ and set $\mathscr{P}(G, \mu):=\left\{\nu \in X: \nu_{\mathrm{G} \text {-dom }} \stackrel{G}{\leq} \mu\right\}$, where $\nu_{\mathrm{G}-\mathrm{dom}}$ stands for the unique element in $X$ that is in the Weyl orbit $W(\nu)$ and is $G$-dominant. Similarly, we set $\mathscr{P}(H, \mu):=\left\{\nu \in Y: \nu_{\mathrm{H} \text {-dom }} \stackrel{H}{\leq} \mu\right\}$, where $\nu_{\mathrm{H} \text {-dom }}$ stands for the unique element in $Y$ that is in the Weyl orbit $W_{H}(\nu)$ and is $H$-dominant. Then we have that

$$
\mathscr{P}(H, \mu)=Y \cap \mathscr{P}(G, \mu) .
$$

Proof. - Since $\mu$ is in $Y$, the result is immediate from Lemmas 4.3 and 4.4.

$4^{\mathrm{e}}$ SÉRIE - TOME $43-2010-\mathrm{N}^{\circ} 6$ 
Lemma 4.6. - We have the following commutative diagram where the vertical maps are the obvious projections

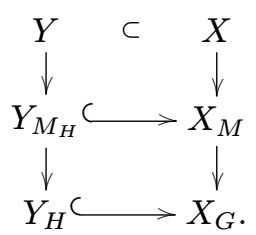

Proof. - We only need to explain why the horizontal maps are (natural) inclusions. This is clear for the first map. For the third map, recall from Remark 4.2 that the coroots corresponding to the simple roots for $\left(T^{\theta}, H\right)$ are $N\left(\alpha^{\vee}\right)$, where $\alpha$ varies through $\mathscr{R}$. This implies that the coroot lattice for $H$ is the intersection of $Y$ with the coroot lattice for $G$, and thus the third map is an inclusion.

Now we will prove that the second map is also an inclusion, with the proof being almost identical to that of the similar fact for the third map. Similar to Remark 4.2, because of condition ( $\dagger$ ), we have that the coroot lattice for $T^{\theta}$ in $M_{H}=M^{\theta}$ has a $\mathbb{Z}$-basis consisting of $N\left(\alpha^{\vee}\right)$, where $\alpha$ varies through a set of representatives for orbits of $\theta$ on $\Delta_{M}$, and where $\Delta_{M}$ is the set of the simple roots for $M$. This implies that the coroot lattice for $T^{\theta}$ in $M_{H}=M^{\theta}$ is just the intersection of $Y$ with the coroot lattice for $T$ in $M$. This ensures that the second horizontal map is injective. That the diagram is commutative follows directly from the definitions of the maps involved.

We now prove Proposition 4.1:

Proof. - It is clear that the left-hand side is contained in the right-hand side. The point is to show that the converse is true as well. Let $\nu \in Y_{M_{H}}$ be an element of the set appearing on the right-hand side in Proposition 4.1. We may assume that $\nu$ is $H$-dominant in $\mathfrak{b}_{M_{H}}$ (otherwise we could pick some other Borel subgroup $B^{\theta}$ in $H$ with respect to which $\nu$ is $H$-dominant). Thus we have the following important properties for $\nu \in Y_{M_{H}}: \nu$ is $H$-dominant, $\nu \stackrel{H}{\leq} \mu$, and $\nu$ and $\mu$ have the same image in $Y_{H}$.

Using lemmas 4.3, 4.4, and 4.6 we see that: $\nu$ is $G$-dominant, $\nu \stackrel{G}{\leq} \mu$, and $\nu$ and $\mu$ have the same image in $X_{G}$. (Using Lemma 4.6, we are viewing $\nu$ as an element in $X_{M}$.) Let $\tilde{\nu} \in X$ be the unique $M$-dominant, $M$-minuscule representative of $\nu$. The results of Section 1 guarantee that $\tilde{\nu} \in \mathscr{P}(G, \mu)$. Then $\theta(\tilde{\nu})$ is the unique $M$-dominant, $M$-minuscule representative of $\theta(\nu)=\nu$. So $\theta(\tilde{\nu})=\tilde{\nu}$, in other words $\tilde{\nu} \in Y$. Using Lemma 4.5 we see that, since $\tilde{\nu}$ lies in both $Y$ and $\mathscr{P}(G, \mu)$, it also lies in $\mathscr{P}(H, \mu)$. We already know that $\nu$ and $\mu$ have the same image in $Y_{H}$, and since $\tilde{\nu}$ evidently maps to $\nu$, we have that $\nu$ is an element of $\psi_{M_{H}}\left(\mathscr{P}_{\mu, H}\right)$, thus concluding the proof of our proposition.

\section{The case of quasi-split groups}

We now work with groups that are quasi-split. Let us fix the notation, since it is slightly different from that of the introduction. Let $F$ be a finite extension of $\mathbb{Q}_{p}$ with uniformizing element $\pi$, and let $L$ be the completion of the maximal unramified extension of $F$ in some algebraic closure of $F$. Denote by $\mathfrak{o}_{F}$, resp. $\mathfrak{o}_{L}$, the ring of integers in $F$, resp. $L$, and by $\sigma$ the 
Frobenius automorphism of $L$ over $F$. Let $G$ be a connected reductive group that is quasisplit over $F$ and split over $L$. Let $A$ be a maximal split torus in $G$, and $T$ its centralizer. Let $B=T U$ be a Borel subgroup of $G$, containing $T$ and $U$ the unipotent radical of $B$. Let $P=M N$ be a parabolic subgroup containing $B$, with $M \supset T$ and $N$ the unipotent radical of $P$. Suppose that all of the above groups are defined over $\mathfrak{o}_{F}$.

The definition of affine Deligne-Lusztig varieties remains the same as in the split case:

$$
X_{\mu}^{G}(b):=\left\{x \in G(L) / G\left(\mathfrak{o}_{L}\right): x^{-1} b \sigma(x) \in G\left(\mathfrak{o}_{L}\right) \mu(\pi) G\left(\mathfrak{o}_{L}\right)\right\},
$$

with $\mu \in X_{*}(T)$ dominant and $b \in M(L)$.

Let $X_{M}$ denote the quotient of the cocharacter lattice $X_{*}(T)$ of $T$ by the coroot lattice for $M$. The Frobenius automorphism $\sigma$ acts on $X_{M}$, and we denote by $Y_{M}$ the coinvariants of this action, i.e., $Y_{M}:=X_{M} /(1-\sigma) X_{M}$. Write $Y$ for the coinvariants of $X_{*}(T)$, and note that we have the following commutative diagram

$$
\begin{array}{ccc}
X_{*}(T) & \rightarrow & X_{M} \\
\downarrow & & \downarrow \\
Y & \rightarrow & Y_{M}
\end{array}
$$

where all the maps are surjective. We denote the map $X_{*}(T) \rightarrow Y$ by $\rho$. We write $\psi$ for the map $Y \rightarrow Y_{M}$ from the above diagram, and then write $\phi: X_{*}(T) \rightarrow Y_{M}$ for the composition $\psi \circ \rho$.

Denote by $\stackrel{P}{\preceq}$ the partial ordering on $Y_{M}$ defined as follows: For $y_{1}, y_{2} \in Y_{M}$, we write $y_{1} \stackrel{P}{\preceq} y_{2}$ if $y_{2}-y_{1}$ is a nonnegative integral linear combination of the images in $Y_{M}$ of the coroots $\left\{\alpha_{j}^{\vee}: j \in J\right\}$ corresponding to simple roots $\left\{\alpha_{j}: j \in J\right\}$ of $T$ in $N$.

Similarly to the Kottwitz maps in the split case from the Introduction, we again have such maps in the quasi-split case, $w_{G}: G(L) \rightarrow X_{G}$ and $w_{M}: M(L) \rightarrow X_{M}$. The latter induces a map $\kappa_{M}: B(M) \rightarrow Y_{M}$ (see [9] for the precise definition), where $B(M)$ stands for the $\sigma$-conjugacy classes in $M(L)$.

Similar to Theorem 1.1 in the case of split groups, we have the following result:

Theorem 5.1. - Let $\mu \in X_{*}(T)$ be dominant and let $b \in M(L)$ be a basic element such that $\kappa_{M}(b)$ lies in $Y_{M}^{+}$. Then $X_{\mu}^{G}(b)$ is non-empty if and only if $\kappa_{M}(b) \stackrel{P}{\preceq} \mu$.

One implication, namely that $X_{\mu}^{G}(b)$ being non-empty implies $\kappa_{M}(b) \stackrel{P}{\preceq} \mu$, is the grouptheoretic version of Mazur's Inequality and a proof of this fact can be found in [10, Theorem 1.1, part (1)]. For the converse, Kottwitz and Rapoport (cf. [10, §4.3]) showed that it follows from Theorem 5.2 below, which they conjectured to be true. To state their conjecture, we need some more notation.

We fix a dominant element $\mu \in X_{*}(T)$, and, as in the introduction, we define the set $\mathscr{P}_{\mu}:=\left\{\nu \in X_{*}(T): \nu=\mu\right.$ in $\left.X_{G}, \nu \in \operatorname{Conv}(W \mu)\right\}$, where $X_{G}$ is the quotient of $X_{*}(T)$ by the coroot lattice for $G$, and $\operatorname{Conv}(W \mu)$ stands for the convex hull in $X_{*}(T) \otimes_{\mathbb{Z}} \mathbb{R}$ of the Weyl group orbit of $\mu$. Write $\mathscr{P}_{\mu, M}$ for the image of $\mathscr{P}_{\mu}$ under the map $\phi: X \rightarrow Y_{M}$.

$4^{\mathrm{e}}$ SÉRIE - TOME $43-2010-\mathrm{N}^{\mathrm{o}} 6$ 
Let $A_{P}$ be the maximal split torus in the center of $M$ and let $\mathfrak{a}_{P}:=X_{*}\left(A_{P}\right) \otimes_{\mathbb{Z}} \mathbb{R}$, where the last space is viewed as a subspace of $X_{*}(T) \otimes_{\mathbb{Z}} \mathbb{R}$. Identifying $Y_{M} \otimes_{\mathbb{Z}} \mathbb{R}$ with $\mathfrak{a}_{P}$, we write $Y_{M}^{+}$for the subset of $Y_{M}$ consisting of elements whose images in $\mathfrak{a}_{P}$ lie in the set

$$
\left\{x \in \mathfrak{a}_{P}:\langle\alpha, x\rangle>0, \text { for all roots } \alpha \text { of } A_{P} \text { in } N\right\} .
$$

Theorem 5.1 follows from the following

Theorem 5.2 (Kottwitz-Rapoport Conjecture; quasi-split case)

Let $\mu \in X_{*}(T)$ be dominant and $\nu_{M} \in Y_{M}^{+}$. The following are equivalent:

(i) $\nu_{M} \stackrel{P}{\preceq} \mu$

(ii) $\nu_{M} \in \mathscr{P}_{\mu, M}$.

(In the condition (i) above we consider $\mu$ as an element of $Y_{M}$.) One sees immediately that (ii) implies (i). The point is to prove that (i) implies (ii). We give a proof of this implication below.

In her Ph.D. thesis [13], Lucarelli proved Theorem 5.2 for unitary groups of rank 3, 4, and 5. Many of her arguments are general and apply to other groups, however, so we will use her ideas and exposition. The crucial added ingredient here is the use of a lemma of Stembridge and of Proposition 2.5.

\subsection{Proof of Theorem 5.2}

Suppose that $\nu_{M} \in Y_{M}^{+}$and that $\nu_{M} \stackrel{P}{\preceq} \mu$, where $\mu \in X_{*}(T)$ is dominant. We would like to prove that there exists an element $\nu \in \mathscr{P}_{\mu}$ such that $\nu \mapsto \nu_{M}$ under the map $\phi: X \rightarrow Y_{M}$. For this purpose, define

$$
\mathscr{P}_{\rho(\mu)}^{\prime}:=\left\{y \in Y: \text { (i) } y \text { and } \rho(\mu) \text { have same image in } Y_{G} \text {, (ii) } y \in \operatorname{Conv}\left(W^{\prime} \rho(\mu)\right)\right\},
$$

where $W^{\prime}$ is the Weyl group associated with $Y$, i.e., the relative Weyl group $N(A)(F) / T(F)$, and $\operatorname{Conv}\left(W^{\prime} \rho(\mu)\right)$ is the convex hull in $Y \otimes_{\mathbb{Z}} \mathbb{R}$ of the orbit $W^{\prime} \rho(\mu)$. Then, since we know that Theorem 5.2 is true for all the corresponding root systems of reduced and non-reduced type (see Remark 5.3 below), we can find $\rho(\nu) \in \mathscr{P}_{\rho(\mu)}^{\prime}$ such that $\psi(\rho(\nu))=\nu_{M}$. Thus it is sufficient to prove that the image of $\mathscr{P}_{\mu}$ under the map $\rho: X_{*}(T) \rightarrow Y$ equals $\mathscr{P}_{\rho(\mu)}^{\prime}$.

REMARK 5.3. - In the split case, since we did not need it there, we did not consider the root system of type $B C_{n}$, the only non-reduced irreducible root system. However, one can deduce Theorem 1.2 for $B C_{n}$ through the process of folding (the root system $A_{2 n}$ ), where one no longer assumes condition $(\dagger)$ from the previous section.

We first show that $\rho\left(\mathscr{P}_{\mu}\right) \subset \mathscr{P}_{\rho(\mu)}^{\prime}$. Suppose that $x \in \mathscr{P}_{\mu}$. Then $x$ has the same image in $X_{G}$ as $\mu$, under the canonical map $X_{*}(T) \rightarrow X_{G}$. Hence $\rho(x)$ and $\rho(\mu)$ have the same image in $Y_{G}$. So, it suffices to prove that $x \in \operatorname{Conv}(W \mu)$ implies $\rho(x) \in \operatorname{Conv}\left(W^{\prime} \rho(\mu)\right)$. For this, we will use two easy facts (whose proofs are omitted):

(a) If $x$ is dominant for $X_{*}(T)$, then $\rho(x)$ is dominant for $Y$, and

(b) If $x \stackrel{!}{\geq} 0$ for $X_{*}(T)$, then $\rho(x) \stackrel{P}{\succeq} 0$ for $Y$. 
(Here $\stackrel{!}{\geq}$ denotes the usual partial ordering in $X_{*}(T)$, where $x_{1} \stackrel{!}{\geq} x_{2}$ means that $x_{1}-x_{2}$ is a nonnegative integer linear combination of simple coroots of $T$ in $N$.)

From $x \in \operatorname{Conv}(W \mu)$ and $\mu$ being dominant, we get that $w x \stackrel{!}{\leq} \mu$ for all $w \in W$, and thus $w^{\prime} x \stackrel{!}{\leq} \mu$ for all $w^{\prime} \in W^{\prime}$, since we can regard $W^{\prime}$ as a subgroup of $W$. Using (a) and (b) we then get that $\rho(\mu)$ is dominant and that $\rho\left(w^{\prime} x\right) \stackrel{P}{\preceq} \rho(\mu)$ for all $w^{\prime} \in W^{\prime}$. But the action of $W^{\prime}$ commutes with $\rho$, so we have $w^{\prime} \rho(x) \stackrel{P}{\preceq} \rho(\mu)$ for all $w^{\prime} \in W^{\prime}$, and thus $\rho(x) \in \operatorname{Conv}\left(W^{\prime} \rho(\mu)\right)$, since $\rho(\mu)$ is dominant. This shows that $x \in \operatorname{Conv}(W \mu)$ implies $\rho(x) \in \operatorname{Conv}\left(W^{\prime} \rho(\mu)\right)$, as desired.

Now that we know that $\rho\left(\mathscr{P}_{\mu}\right) \subset \mathscr{P}_{\rho(\mu)}^{\prime}$, we would like to prove the other inclusion. Suppose that $\nu^{\prime} \in \mathscr{P}_{\rho(\mu)}^{\prime}$. Without loss of generality we may assume that $\nu^{\prime}$ is dominant. Then we have that $\nu^{\prime} \stackrel{P}{\preceq} \rho(\mu)$. Due to the transitive property of $\stackrel{P}{\preceq}$, we only need to consider the case when $\rho(\mu) \operatorname{covers} \nu^{\prime}$. Recall that we say that $\rho(\mu) \operatorname{covers} \nu^{\prime}$ if for any $v$ with $\nu^{\prime} \stackrel{P}{\preceq} v \stackrel{P}{\preceq}$ $\rho(\mu)$ we have that $v=\nu^{\prime}$ or $v=\rho(\mu)$. Suppose, therefore, that $\rho(\mu)$ covers $\nu^{\prime}$. Then using a lemma of Stembridge ([21, Cor. 2.7]; see also [17, Lemma 2.3], for an alternative proof, due to Waldspurger, of this result) we can conclude that there exists a positive coroot $\beta^{\vee}$ such that $\nu^{\prime}=\rho(\mu)-\beta^{\vee}$. Thus, in order to prove Theorem 5.2, it suffices to show the following:

Proposition 5.4. - There exists an element $\nu \in \mathscr{P}_{\mu}$ such that $\rho(\nu)=\rho(\mu)-\beta^{\vee}$.

Proof. - Denote by $R$ the root system formed by the coroots of $G$ in $X_{*}(T)$, and by $R^{\prime}$ the one obtained by taking the image of $R$ under $\rho$. More precisely, the coroots of $R^{\prime}$ are obtained by taking the images of the coroots of $R$ under the map $\rho$.

Remark 5.5. - Note that we do not get $R^{\prime}$ from $R$ by folding, which would amount to taking invariants under the automorphism $\sigma$. Rather, we are cofolding the root system $R$ to get $R^{\prime}$, i.e., we are taking the coinvariants under the action of $\sigma$. For example, folding $A_{2 n-1}$ would yield $B_{n}$, but cofolding $A_{2 n-1}$ yields $C_{n}$. Also, we remark again that we are working with coroots and not roots.

One sees immediately that there exists a (positive) $\operatorname{coroot} \gamma^{\vee}$ such that $\rho\left(\gamma^{\vee}\right)=\beta^{\vee}$ and $\langle\mu, \gamma\rangle \geq 1$. Indeed, since $\mu$ is dominant, we have that for all coroots $\gamma^{\vee}$ with the property that $\rho\left(\gamma^{\vee}\right)=\beta^{\vee}$, we must have that $\langle\mu, \gamma\rangle \geq 0$. If for all these $\gamma$ we had $\langle\mu, \gamma\rangle=0$, then we would get $\langle\rho(\mu), \beta\rangle=0$. But this would give $\left\langle\nu^{\prime}, \beta\right\rangle=-2$, contradicting the assumed dominance of $\nu^{\prime}$.

Now we put $\nu:=\mu-\gamma^{\vee}$. Denote by $\omega_{j}, j \in J$, the set of fundamental weights, where $\omega_{j}$ corresponds to the simple coroot $\alpha_{j}^{\vee}$, in $X_{*}(T)$. Recall the definition of the cone

$$
C_{\mu}^{+}:=\left\{u \in X_{*}(T) \otimes_{\mathbb{Z}} \mathbb{R} \mid\left\langle u, \omega_{j}\right\rangle \leq\left\langle\mu, \omega_{j}\right\rangle\right\}
$$

and see immediately that $\nu \in C_{\mu}^{+}$. Using Proposition 2.3, we see that Proposition 5.4 follows if we show that there exists an element $w \in W$ such that $w$ is $\nu$-minuscule and $w(\nu)$ is dominant. But, from Proposition 2.5 we have that this is the case if and only if

$$
\langle\nu, \alpha\rangle \geq-1, \text { for all } \alpha \in R^{+} .
$$

It remains to prove that these inequalities hold in our case.

$4^{\text {e }}$ SÉRIE - TOME $43-2010-\mathrm{N}^{\mathrm{o}} 6$ 
Recall that $\nu=\mu-\gamma^{\vee}$, where $\gamma^{\vee}$ is a positive coroot such that $\langle\mu, \gamma\rangle \geq 1$. If $\alpha \in R^{+} \backslash\{\gamma\}$, since $R$ is simply-laced, it is well known that we must have $\left\langle\gamma^{\vee}, \alpha\right\rangle \leq 1$. Therefore $\langle\nu, \alpha\rangle=\langle\mu, \alpha\rangle-\left\langle\gamma^{\vee}, \alpha\right\rangle \geq 0-1=-1$. For $\alpha=\gamma$, we have $\langle\nu, \gamma\rangle=\langle\mu, \gamma\rangle-\left\langle\gamma^{\vee}, \gamma\right\rangle \geq$ $1-2=-1$. This concludes the proof of Proposition 5.4 and therefore of Theorem 5.2.

\section{REFERENCES}

[1] J. Arthur, A local trace formula, Publ. Math. I.H.É.S. 73 (1991), 5-96.

[2] N. Bourbaki, Lie groups and Lie algebras. Chapters 4-6, Elements of Mathematics, Springer, 2002.

[3] N. BourbaKi, Lie groups and Lie algebras, Chapters 7-9, Elements of Mathematics, Springer, 2005.

[4] Q. R. GASHI, A vanishing result for toric varieties associated with root systems, Albanian J. Math. 1 (2007), 235-244.

[5] Q. R. GASHI, Vanishing results for toric varieties associated to $\mathrm{GL}_{n}$ and $\mathrm{G}_{2}$, Transform. Groups 13 (2008), 149-171.

[6] Q. R. Gashi, T. Schedler, On dominance and minuscule Weyl group elements, preprint arXiv:0908.1091.

[7] M. Görtz, T. J. Haines, R. E. Kottwitz, D. C. Reuman, Affine Deligne-Lusztig varieties in affine flag varieties, preprint arXiv:0805.0045.

[8] U. Görtz, T. J. Haines, R. E. Kottwitz, D. C. Reuman, Dimensions of some affine Deligne-Lusztig varieties, Ann. Sci. École Norm. Sup. 39 (2006), 467-511.

[9] R. E. Kоттwitz, Isocrystals with additional structure, Compositio Math. 56 (1985), 201-220.

[10] R. E. Коттwitz, On the Hodge-Newton decomposition for split groups, Int. Math. Res. Not. 2003 (2003), 1433-1447.

[11] R. E. Kottwitz, M. Rapoport, On the existence of F-crystals, Comment. Math. Helv. 78 (2003), 153-184.

[12] C. Lucarelli, A converse to Mazur's inequality for split classical groups, J. Inst. Math. Jussieu 3 (2004), 165-183.

[13] C. Lucarelli, A converse to Mazur's inequality for split classical groups, Ph.D. Thesis, University of Chicago, 2004.

[14] B. Mazur, Frobenius and the Hodge filtration, Bull. Amer. Math. Soc. 78 (1972), 653667.

[15] B. Mazur, Frobenius and the Hodge filtration (estimates), Ann. of Math. 98 (1973), 58-95.

[16] S. Mozes, Reflection processes on graphs and Weyl groups, J. Combin. Theory Ser. A 53 (1990), 128-142.

[17] M. Rapoport, A positivity property of the Satake isomorphism, Manuscripta Math. 101 (2000), 153-166.

[18] M. RAPOPORT, A guide to the reduction modulo $p$ of Shimura varieties, Astérisque 298 (2005), 271-318. 
[19] M. Rapoport, M. Richartz, On the classification and specialization of $F$-isocrystals with additional structure, Compositio Math. 103 (1996), 153-181.

[20] T. A. Springer, Linear algebraic groups, $2^{\text {nd }}$ ed., Progress in Mathematics, Birkhäuser, 2006.

[21] J. R. Stembridge, The partial order of dominant weights, Adv. Math. 136 (1998), 340 364.

[22] J. R. Stembridge, Minuscule elements of Weyl groups, J. Algebra 235 (2001), 722-743.

[23] E. Viehmann, The dimension of some affine Deligne-Lusztig varieties, Ann. Sci. École Norm. Sup. 39 (2006), 513-526.

[24] J.-P. Wintenberger, Existence de $F$-cristaux avec structures supplémentaires, $A d v$. Math. 190 (2005), 196-224.

\author{
Qëndrim R. GaSHI \\ Institut des Hautes Études Scientifiques \\ Le Bois-Marie \\ 35 , route de Chartres \\ 91440 Bures-sur-Yvette, France \\ and \\ University of Prishtina \\ SFHMN, Departamenti i Matematikes \\ rr. "Nena Tereze" \\ Pristina, 10000, UNMIK Kosovo \\ E-mail: gashi@ihes.fr
}

